\title{
A Non-Contact Capacitance Type Level Transducer for Liquid Characterization
}

\author{
Sheroz Khan, Khalifa, Kyaw Kyaw Htike@ Muhammad Ali, A. H. M. Zahirul Alam, Mohd \\ Rafiqul Islam, Othman O. Khalifa, Saman S. Khan \\ ${ }^{1}$ Internaitonal Islamic University Malaysia \\ Email:sheroz@iiu.edu.my
}

\begin{abstract}
The liquid properties such as buoyancy, pressure at $a$ depth, relative electrical permittivity, electrical conductivity, thermal conductivity, absorption of radiation, liquid surface reflection of sound or light waves, are used to design the different types of liquid level transducers for liquid level measurement in any process industry. The contact-type level-sensing transducers have the disadvantage that their characteristic properties may change due to physical or chemical reaction between the liquid and the probing material, and hence may affect accuracy besides their life time.

The non-contact-type level-sensing probes may have longer life period, but they are comparatively costly and require various environmental and experimental precautionary measures when being used.

In this paper, a low-cost non-contact capacitance type liquid level measuring technique has been designed and used for liquid identification objectives. The results obtained are very much consistent with the theoretical derivations.
\end{abstract}

\section{INTRODUCTION}

The liquid properties like buoyancy, pressure at a depth, relative electrical permittivity, electrical conductivity, thermal conductivity, absorption of radiation, liquid surface reflection of sound or light waves, etc., are related with the liquid level and are used to design liquid level Liquid level transducers in any process industry [1-4]. In the contact-type leveltransducers level sensing probes are used which have the inaccuracy disadvantage besides limited life time as their characteristic properties may change due to the physical and chemical reaction between the liquid and the probe material [7-9].
The non-contact-type level-sensing probes unlike may have longer life period, are comparatively costly and require various environmental and experimental precautions during the measurement. The optical fiber liquid level transducer is a contact-type transducer, which produces optical pulses when the liquid level moves along the cladding and un-cladding zones of the optical fiber. This transducer has a further advantage that it can be used with intrinsic safety in explosive or inflammable liquids [13] [14].

However, this type of measuring system is much costlier than the other similar instruments. In this paper, a low-cost new type of non-contact capacitance type liquid level measuring technique has been designed. In the conventional non-contact capacitancetype liquid level transducer the air column between the conducting liquid level and the sensing electrode of a capacitor is used as the dielectric. This technique appears to suffer from the error due to the frequent changes of the dielectric properties of air as well as due to the low value of permittivity of air and, thus, appears to have limited applications.

In the technique as described in this paper, the material of a uniform right circular cylinder made of polyvinyl chloride (PVC), is used as a dielectric of a cylindrical capacitor. Now, the change in capacitance of a capacitive transducer due to a change in process variable is generally very small [14] [15] [16].

In the present paper, an operational-amplifier (opamp)-based modified bridge network is used to measure changes in capacitance of a proposed levelsensing probe, where the effect of stray capacitance between the output leads of the bridge network is minimized. The experimental results are found to have good repeatability, linearity, and resolution.

\section{Current Status of Technology}

Capacitive transducer measurement procedure requires a probe to sense changes in capacitance as a 
measure of a physical quantity, some driver electronics to convert these changes in capacitance into voltage changes, and a device to indicate or record resulting voltage changes.

The probe geometry, sensing area size, and mechanical construction affect range, accuracy, and stability. The performance of the driver electronics is a primary factor in determining the resolution of the system. The voltage measuring device is the final link in the system, and must be properly selected for a specific application.

\section{EXPERIMENTAL SETUP AND METHOdOLOGY}

The experimental setup of this work consists of a custom made water container with a circular tube running alongside from bottom-to-top as shown in Figure 1. Among the extra hardware tools used are digital and analogue oscilloscopes.

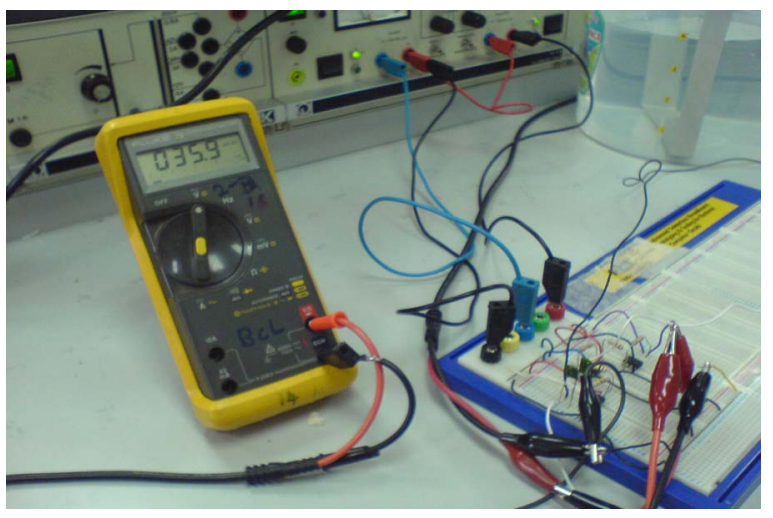

Figure 1. The Laboratory Experimental Setup

In this work experimental data is obtained for the following:

1. Normal tap water

2. Salt-dissolved water (2 spoons of sugar)

3. Salt-dissolved water (4 spoons of sugar)

4. Sugar-dissolved water (2 spoons of sugar)

5. Sugar-dissolved water (4 spoons of sugar)

The container makes a variable capacitance depending upon the level of the liquid within it, while the rest of the electronic is as shown in Fig. 2.

The measuring circuit is making use of a bridge circuit, where the unknown capacitance is found by balancing the bridge with the extra capacitance. The transfer function $\mathrm{H}(\mathrm{s})$ of the circuit is made from the lower and upper part of the circuit. The lower is further made from two transfer functions in series, and so are they multiplied to calculate the total transfer of the lower part. The over all transfer function is hence calculated through a sum of the two transfer functions.

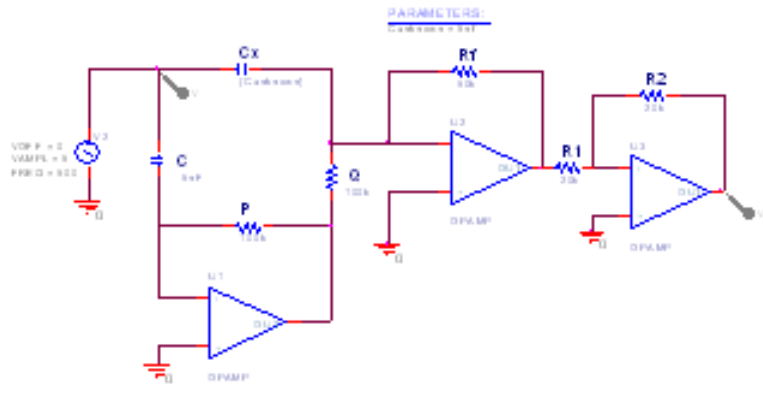

Figure 2. Basic measuring and electronic circuit

$$
\begin{gathered}
H(s)_{L 1}=-\frac{P}{\frac{1}{s C}}=-s C P \quad H(s)_{L 2}=-\frac{R_{f}}{Q} \\
H(s)_{L}=H(s)_{L 1} H(s)_{L 2}=s C P \frac{R_{f}}{Q} \ldots \ldots . . .
\end{gathered}
$$

For a balanced bridge

$C P=C_{o} Q$, so

$H(s)_{L}=H(s)_{L 1} H(s)_{L 2}=s C_{o} R_{f}$

$H(s)_{H}=-s C_{x} R_{f}=-s C_{o} R_{f}-s \Delta C R$

Where $C x=C o+\Delta C$, residual capacitance and change in capacitance due liquid level change Hence transfer function until the end of $\mathrm{U} 2$ is $H(s)=H_{L}+H_{H}=s C_{o} R_{f}-s C_{o} R_{f}-s \Delta C R_{f}=-s \Delta C R_{f} \ldots \ldots$ (3 )

The overall transfer function including the last leg of the circuit including $\mathrm{U} 3$ is given by

$$
H(s)_{\text {TOTAL }}=-\frac{R_{2}}{R_{1}}\left(-s \Delta C R_{f}\right)=\frac{R_{2}}{R_{1}} s \Delta C R_{f}
$$

The bridge is balanced for the original residual capacitance without the liquid, hence a liquid-level increase beyond which will lead to an output voltage given by

$$
V_{o}=V_{\text {in }} H(s)_{\text {TOTAL }}=\left(\frac{R_{2}}{R_{1}} s \Delta C R_{f}\right) V_{\text {in }}
$$

The increase in output voltage is proportional to changes in capacitance and if the input voltage applied is constant with a stable frequency. The PSPICE simulation output voltage for five values of capacitance is as shown in Fig. 3, where the input voltage is sinusoidal and the output is inverted and advanced by $90^{\circ}$ as well proportionately increasing capacitance values. 


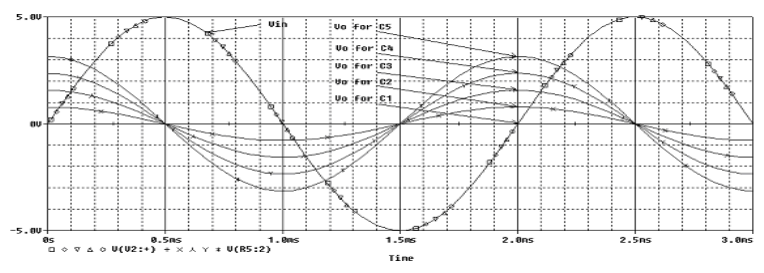

Figure 3. PSpice simulation of the output voltage waveforms

A modified version of the shown in Fig. 4, where the source is directly applied to the positive of on op amp (U2) and through a divider (R7, R8) to another op amp. The bridge under balanced condition will reproduce the input at the input terminal to R3 and hence to op amp U1. Thus the op amp U3 acts as a differentiator, with the input coming from the bridge circuit higher in value than the one coming form the divider circuit, hence giving to a voltage value in phase with the input for a balanced bridge circuit.

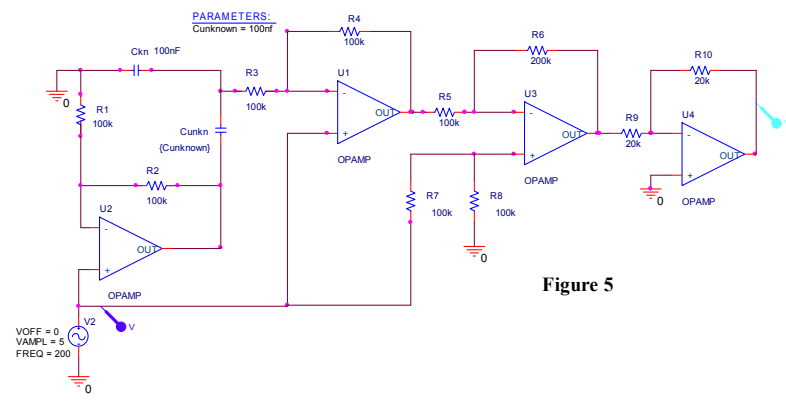

Figure 4. The second amplification circuit

The output voltage in this case is independent of frequency, inverse proportional to the change in capacitance and is in phase with the input voltage as shown in Figure 5.

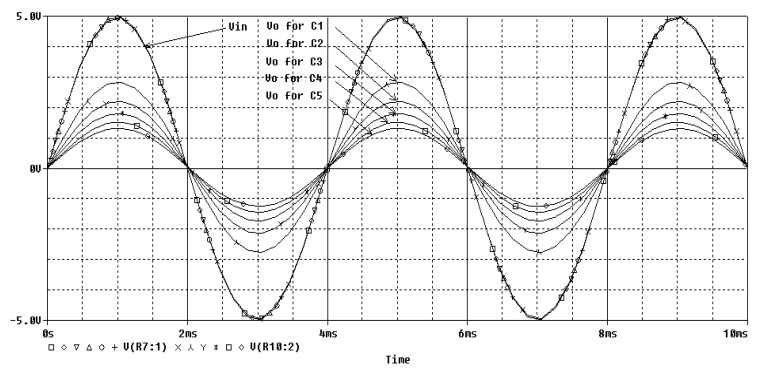

Figure 5. PSpice simulation of modified output voltage waveforms

The insulating material of the sensing cylinder is taken as the dielectric of the cylindrical capacitor, whereas the conducting liquid column inside the cylinder is taken as the grounded electrode of the capacitor because the liquid is generally stored in a metallic grounded vessel. Thus, the vessel itself may be taken as one electrode of the cylindrical capacitor, whereas an outer double-layer non-inductively wound short-circulated coil may be taken as the other electrode of the capacitor. Inasmuch as the coil is short-circuited, all the turns will be at the same potential, and the inter-winding capacitance and interlayer capacitance of the coil may be assumed to be negligible. Moreover, the short-circuited outer layer coil and the short-circuited inner layer coil have the same conducting material, and hence, the effect of the inner layer coil on the capacitance of the whole twolayer coil with respect to the liquid column may be ignored. Thus, the capacitance between the liquid column and the short-circuited non-inductively wound two-layer coil may be assumed to be equivalent to the capacitance between the liquid column and only the outer layer of the coil. The cross section of the level sensor is shown in Fig. 1(b). Let the coil be made from a PVC- or enamel-insulated single-strand copper wire of internal conductor radius $r$ with insulation thickness $t$ and the internal and external diameters of the sensing cylinder as $d$ and $D$, respectively. Now, a single-turn coil of conductor radius $r$ of the outer layer may be assumed to be a very small cylinder of height $2 r$, and a cylindrical capacitor will be formed between the coil and the inner conducting liquid column of diameter $d$, which is equal to the inner diameter of the levelsensing cylinder.

\section{RESUltS AND TABULATED DATA}

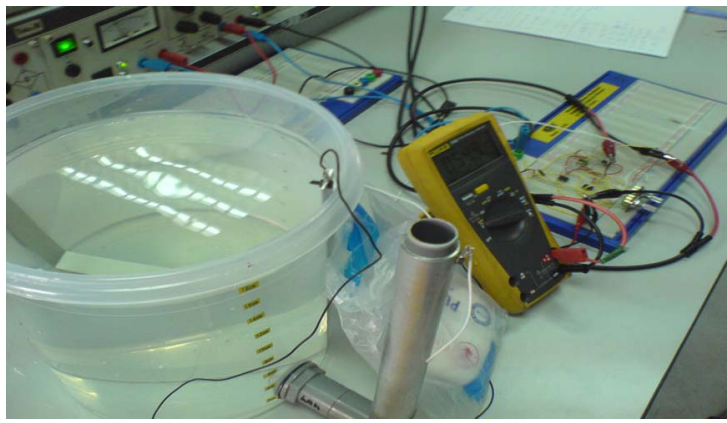

Figure 6. Experimental Set-up Showing Water Container

We have repeated all the measurements to ensure accuracy. Furthermore, for each liquid, we performed increasing and decreasing levels of liquid to estimate the $\%$ error for all the measurements. From this result section, we can observe that both 'increasing' and 'decreasing' curves give almost linear straight lines and the 'increasing' and 'decreasing' curves are almost the same (with opposite slopes). 

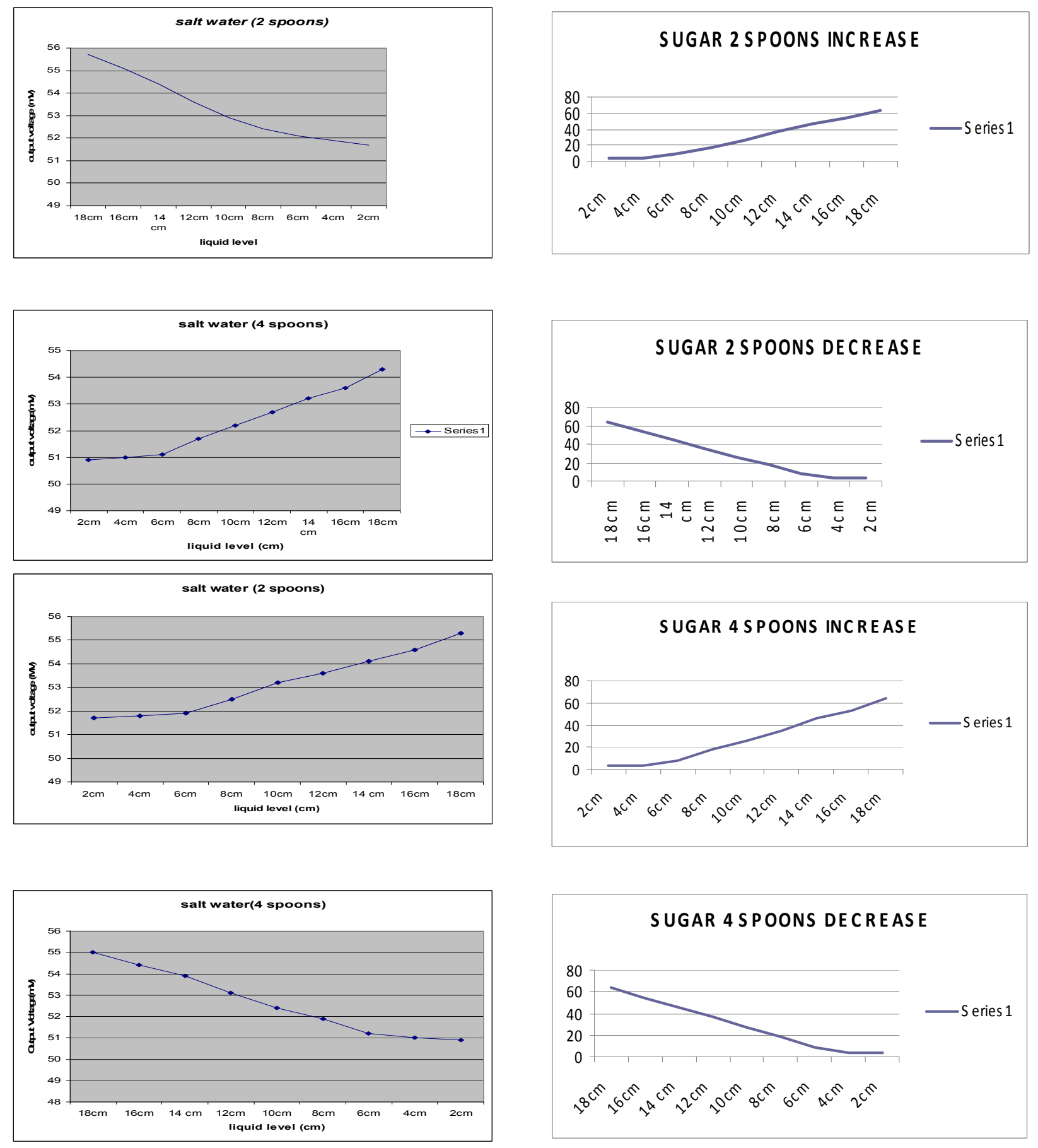


\section{DISCUSSION AND ERROR ANALYSIS}

The results show good linearity and an acceptable level of accuracy in all of the measurements, however, a few critical points need to be mentioned:

- The results follow an approximately horizontal straight line until the liquid level reaches around $6 \mathrm{~cm}$ where the metal casing is connected to plastic water tank, a point where from a good linearity is obtained.

- Even when there is no liquid inside the tank, there is still some small output voltage, which means some residual capacitance is present.

- The new (or the second) circuit appears to give more accurate and consistent data than the old op-amp circuit.

- For all sets of linear graphs obtained, we calculated the estimate of the straight lines representing the experimental data from which we calculated the \% systematic and \% random errors using linear regression formula and Microsoft Excel.

The experimental data is plotted for errors with the calculated are as shown in Fig. 7 (a-h) for various solution of known solvent quantities.

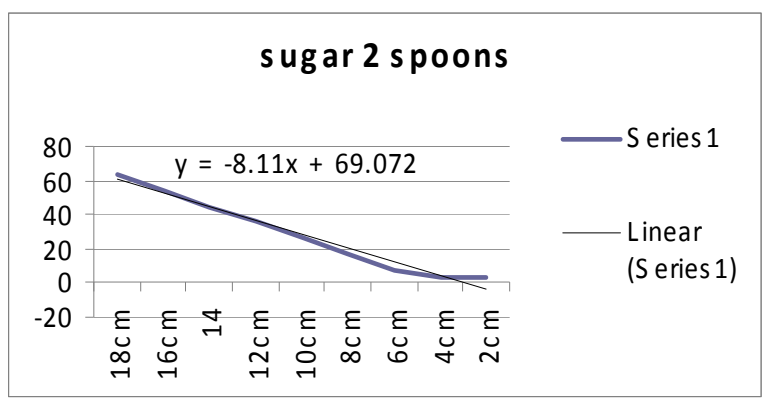

(a)

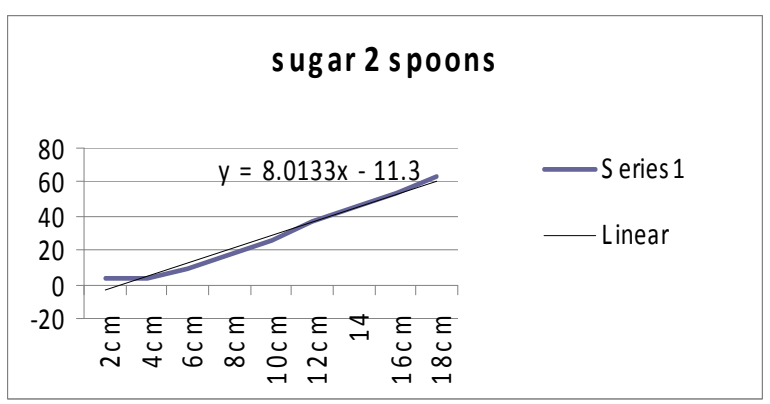

(b)

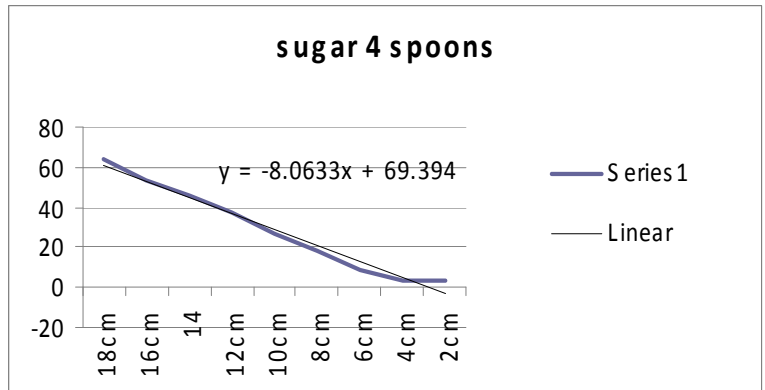

(c)

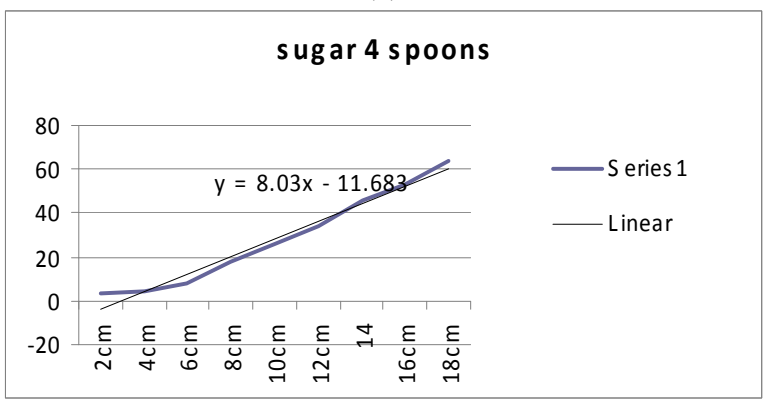

(d)

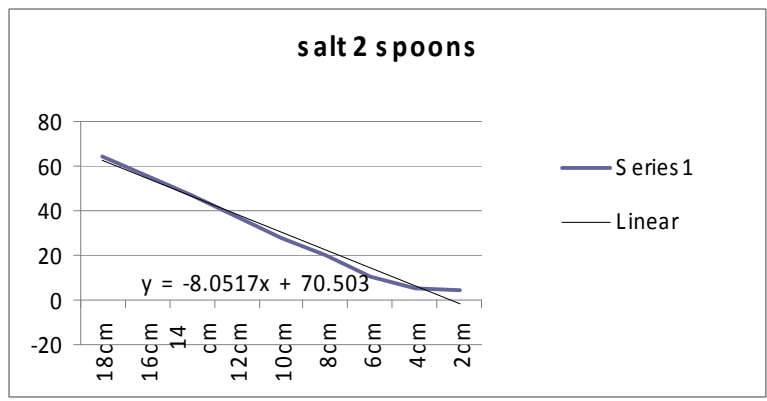

(e)

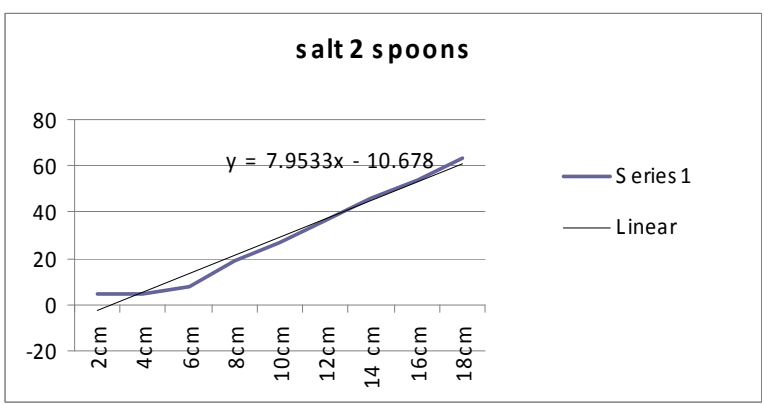

(f) 


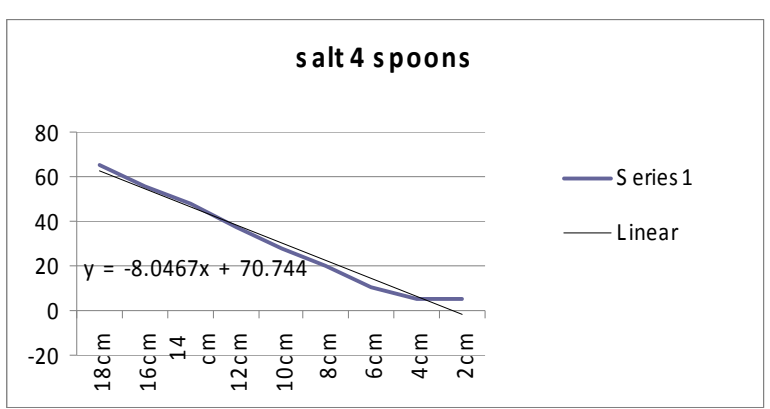

(g)

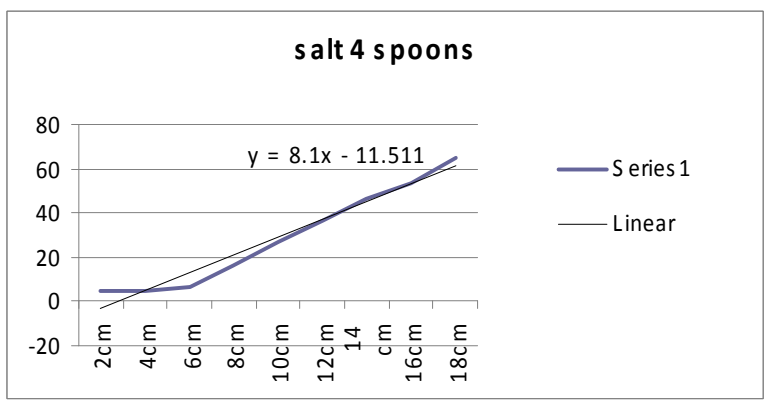

(h)

\section{CONCLUSIONS}

In this work, properties of a low-cost non-contact capacitance-type level transducer are studied and analyzed for various conducting liquid. The liquid level in each experiment was varied in steps under the static condition, and the bridge output voltage of each step was measured by digital $41 / 2$ digit multi-meter and the digital and analogue oscilloscopes and graphically plotted for all the values. The static characteristic curves of the developed. Using linear regression, the data is plotted and percentage error is obtained, and is found to be within tolerable limits. The modified De' Sauty bridge circuit was found to give more stable results than the conventional De' Sauty bridge circuit used in the capacitance-type level probe. Here, it may be mentioned that for the bridge set-up, the circuit common and the storage tank ground should be isolated from each other. Hence, during the design and experimental work, proper care should be taken so that this isolation may not be disturbed by any means; otherwise, the results may be erroneous. To avoid the incidental or accidental short circuiting between these two grounds, a non-inverting-type opamp-based circuit may be used. The life of the level sensor developed is expected to be long compared with other non-contact level gauges because there is no wear and tear of the sensor during operation.

\section{REFERENCES}

[1] A. J. Jaworski, T. Dyakowski, and G. A. Davies, "A capacitance probe for nterface detection in oil and gas extraction plant," Meas. Sci. Technol., ol. 10, no. 3, pp. L15L20, Mar. 1999.

[2] W. R. Yang, M. R. Brant, and M. S. Beck, "A multi-interface level measurement ystem using a segmental capacitance sensor for oil separators," eas. Sci. Technol., vol. 5, no. 9, pp. 11771180, Sep. 1994

[3] S. M. Herang, R. G. Green, A. Plaskowski, and M. S. Beck, "A high requency stray immune capacitance transducer based on the charge transfer rinciple," IEEE Trans. Instrum. Meas., vol. 37, no. 3, pp. 368-373, ep. 1988.

[4] A. H. Falkner, "The use of capacitance in the measurement of angular nd linear displacement," IEEE Trans. Instrum. Meas., vol. 43, no. 6, p. 939-942, Dec. 1994.

[5] G. Betta, A. Pietrosanto, and A. Scaglione, "A Gray code based fiber ptic liquid level transducer," IEEE Trans. Instrum. Meas., vol. 47, no. 1, p. 174-178, Feb. 1998.

[6] , "Microcontroller-based performance enhancement of an optical iber level transducer," IEEE Trans. Instrum. Meas., vol. 47, no. 2, p. 489-493, Apr. 1998.

[7] P. Holmberg, "Automatic balancing of linear AC bridge circuits for capacitive ensor elements," IEEE Trans. Instrum. Meas., vol. 44, no. 3, p. 803-805, Jun. 1995.

[8] D. Marioli, E. Sardini, and A. Taroni, "High accuracy measurement techniques for capacitance transducers," Meas. Sci. Technol., vol. 4, no. 3, pp. 337-343, Mar. 1993.

[9] C. Kolle and P. O'Leary, "Low cost, high precision measurement system for capacitive sensors," Meas. Sci. Technol., vol. 9, no. 3, pp. 510-517, Mar. 1998.

[10] E. O. Doeblin, Measurement System Application and Design, 4th ed. New York: McGraw-Hill, 1990.

[11] B. G. Liptak, Process Measurement and Analysis, 3rd ed. Oxford, U.K.: Butterworth Heinman, 1999.

[12] D. M. Considine, Process Instruments and Control Hand Book, 2nd ed. New York: McGraw-Hill, 1974.

[13] J. P. Bently, Principles of Measurement Systems, 3rd ed. Singapore: Longman Singapore Publishers (Pte) Ltd., 1995.

[14] Level Measurement, p. 27, Dec. 1993. Control Instrum. (UK), Survey Directory.

[15] E.W. Golding and F. C.Widdis, Electrical Measurements and Measuring Instruments, 5th ed. London, U.K.: Pitman, 1963.

[16] A Low-Cost Noncontact Capacitance-Type Level Transducer for a Conducting Liquid IEEE TRANSACTIONS ON INSTRUMENTATION AND MEASUREMENT, VOL. 55, NO. 3, JUNE 2006 